\title{
Effects of Liraglutide on Nonalcoholic Fatty Liver Disease in Patients with Type 2 Diabetes Mellitus: A Systematic Review and Meta-Analysis
}

\author{
Tiantian Song · Yujiao Jia · Zelin Li · Fei Wang · Luping Ren • \\ Shuchun Chen
}

Received: March 22, 2021 / Accepted: May 1, 2021 / Published online: May 17, 2021

(C) The Author(s) 2021

\begin{abstract}
Introduction: Nonalcoholic fatty liver disease (NAFLD) is a common comorbidity of type 2 diabetes mellitus (T2DM), and no approved therapies are currently available. A meta-analysis was performed to investigate the effects of liraglutide on NAFLD in patients with T2DM.

Methods: Medline (via PubMed), Embase (via Elsevier), and the Cochrane Central Register of Controlled Trials (CENTRAL) (via Cochrane Library) from inception to April 2020 were searched. After screening the literature and extracting data, we assessed the risk of bias of the eligible studies. The Cochrane Collaboration's RevMan software program was used for the statistical analysis.
\end{abstract}

Supplementary Information The online version contains supplementary material available at https:// doi.org/10.1007/s13300-021-01072-4.

T. Song · Y. Jia · Z. Li · F. Wang

Graduate School of Hebei Medical University, Shijiazhuang, China

T. Song · Y. Jia · Z. Li · F. Wang · L. Ren · S. Chen Department of Endocrinology, Hebei General Hospital, Shijiazhuang, China

L. Ren $\cdot$ S. Chen $(\bowtie)$

Hebei Key Laboratory of Metabolic Diseases,

Graduate School of Hebei Medical University,

Shijiazhuang, China

e-mail: chenshuc2014@163.com
Results: Eleven trials involving 535 patients were included for the final analysis. Compared to the placebo or control group, liraglutide decreased liver fat (LF) (insulin: mean difference $\mathrm{MD}-2.50,95 \%$ confidence interval $[\mathrm{CI}]-4.30$ to -0.70 ), body mass index (BMI) (placebo: $\mathrm{MD}$ $-1.13,95 \%$ CI -2.03 to -0.23 ; pioglitazone: MD - 4.10, 95\% CI - 6.27 to - 1.93 ; metformin: $\mathrm{MD}-1.07,95 \% \mathrm{CI}-2.06$ to -0.08 ; insulin: $\mathrm{MD}-1.01,95 \% \mathrm{CI}-1.60$ to -0.43 ), lipoproteins, including high-density (insulin: MD - 0.10, 95\% CI - 0.15 to - 0.05) and lowdensity lipoproteins (MD $-0.26,95 \% \mathrm{CI}-0.43$ to - 0.10), glycated hemoglobin A1c (HbA1c) (placebo: $\mathrm{MD}-0.86$; $95 \% \mathrm{CI}-1.22$ to -0.51 ; insulin: $\mathrm{MD}-0.22,95 \% \mathrm{CI}-0.41$ to -0.04$)$, total cholesterol (placebo: MD - 0.34, 95\% CI -0.65 to -0.03 ; metformin: MD $0.09,95 \%$ CI $0.01-0.18$ ), and triglycerides (placebo: MD $-0.29,95 \%$ CI -0.57 to -0.01 ; insulin: MD - 0.80, 95\% CI -1.03 to -0.57$)$. Liraglutide may be associated with increased gastrointestinal reactions compared to pioglitazone.

Conclusion: These findings revealed that liraglutide decreased LF, BMI, lipids, or HbA1c in T2DM patients complicated with NAFLD, indicating its potential therapeutic efficacy. 
Keywords: Liraglutide; Meta-analysis; Nonalcoholic fatty liver disease; Systematic review; Type 2 diabetes mellitus

\section{Key Summary Points}

\section{Why carry out this study?}

Nonalcoholic fatty liver disease (NAFLD) is a common comorbidity of type 2 diabetes mellitus (T2DM), but no approved therapies are yet available for its treatment.

Liraglutide supposedly has the potential to treat this patient population because it can effectively alleviate T2DM and lower adverse events in T2DM patients; however no consensus on its efficacy in this context has been reached.

We performed a systematic review and meta-analysis to comprehensively determine the actual effects of liraglutide on NAFLD in patients with T2DM.

\section{What was learned from the study?}

When compared to placebo or control drugs, the benefits of liraglutide in patients with T2DM complicated with NAFLD include decreasing liver fat, body mass index, lipid levels, and glycated hemoglobin.

Liraglutide may be the preferred treatment option for treating NAFLD in patients with T2DM due to its therapeutic efficacy.

Further studies with a sufficient sample size should be designed to establish the effectiveness and safety of liraglutide for the treatment of NAFLD in patients with T2DM because of the inadequate number of eligible studies and small sample sizes.

\section{DIGITAL FEATURES}

This article is published with digital features, including a summary slide, to facilitate understanding of the article. To view digital features for this article go to https://doi.org/10.6084/ m9.figshare.14519067.

\section{INTRODUCTION}

Type 2 diabetes mellitus (T2DM) is the most common public health problem worldwide [1], with an estimated 415 million confirmed cases in 2015 [2, 3]. It is expected that the number of confirmed cases of T2DM will reach 642 million by 2040 [4]. Studies have revealed a strong relationship between T2DM and nonalcoholic fatty liver disease (NAFLD), with reports suggesting that about $70-90 \%$ of patients with T2DM are likely to suffer from NAFLD $[5,6]$. In turn, NAFLD may become a crucial contributor to liver-related mortality. Liver transplantation during the next 5 years is also expected to increase at an alarming rate in patients with T2DM [7].

The histologic spectrum of NAFLD ranges from simple steatosis to nonalcoholic steatohepatitis (NASH), liver cirrhosis, liver failure, and even hepatocellular carcinoma [8]. It is important to note that patients with NAFLD who progress to NASH are at increased risk of morbidity and mortality related to liver and cardiovascular disease [9-11]. Therefore, it is necessary to prevent and treat NAFLD in order to manage T2DM. Unfortunately, there are currently no approved therapies with beneficial effects for the management of NAFLD in patients with T2DM. Lifestyle modifications, including dietary modifications and weight loss, still play a vital role in the management of NAFLD [12]. Although many studies have explored the role of several pharmacological agents, such as statins, omega-3 polyunsaturated fatty acids, vitamin $\mathrm{E}$, and pioglitazone, in the treatment of NAFLD, few have reported beneficial results [13-17]. In addition, the concerns regarding the side effects and long-term safety profiles of these agents, such as pioglitazone and vitamin $\mathrm{E}$, have limited their use for 
treatment [18]. Bariatric and metabolic sugery (BMS) is one of the most efficient and scientifically successful methods for treating patients with excessive amount of adipose tissue [19], and it has also shown to be a promising strategy for the treatment of obesity, T2DM and comorbidities [20]. However, owing to safety issues, BMS is still not recommended by American Association for the Study of Liver Diseases to specifically treat NAFLD and NASH [21].

Liraglutide is an analog of incretin hormone glucagon-like peptide (GLP-1) that is extensively prescribed for the treatment of T2DM $[22,23]$. Previous studies have revealed an alleviation of T2DM after treatment initiation with liraglutide, which stimulates the secretion of insulin and then promotes glucose metabolism without causing significant hypoglycemia [24]. Liraglutide is also used to lower bodyweight and reduce the risk of cardiovascular events in patients with T2DM $[25,26]$. Given these benefits, several human studies [27-30] have investigated the effects of liraglutide for the treatment of NAFLD in patients with T2DM. However, conflicting findings with regard to the efficacy and safety of liraglutide have limited decision-making [31, 32]. Therefore, this systematic review and meta-analysis was designed to comprehensively determine the efficacy and safety of liraglutide in the treatment of NAFLD in adult patients with T2DM.

\section{METHODS}

This article is based on previously conducted studies and does not contain any new studies with human participants or animals performed by any of the authors.

The framework of the current systematic review and meta-analysis was designed based on the recommendations proposed by the Cochrane Collaboration (CC) [33]. All results were summarized in accordance with the framework issued by the Preferred Reporting Items for Systematic Reviews and Meta-Analysis (PRISMA) statement [34].

\section{Identification of Eligible Literature}

Two independent investigators searched all citations in the Medline (via PubMed), Embase (via Elsevier), and Cochrane Central Register of Controlled Trials (CENTRAL) (via Cochrane library) databases from inception through April 2020. The reference lists of all eligible trials and topic-related reviews were checked manually to identify any potential trial. The relevant studies were searched by combining Medical Subject Headings (MeSH) and text words according to the unique requirements of each individual database [35]. All search strategies of targeted databases are documented in Electronic Supplementary Material (ESM) Table S1.

\section{Selection Criteria}

Randomized controlled trials that compared liraglutide against placebo, pioglitazone, metformin or insulin for the treatment of NAFLD in patients with T2DM were included. Eligible studies had to report at least one of the following outcomes: liver enzymes, including aspartate aminotransferase (AST), alanine aminotransferase (ALT), gamma-glutamyl transferase (GGT), and alkaline phosphatase (ALP); liver histology (measured as liver fat content [LF]); metabolic factors, such as body mass index (BMI), glycated hemoglobin A1c (HbA1c), visceral adipose tissue (VAT), and subcutaneous adipose tissue (SAT); lipid metabolism, including total cholesterol (TC), triglycerides (TG), high-density lipoprotein (HDL), and low-density lipoprotein (LDL); and adverse events (AEs). We defined liver enzymes as the primary outcomes, and the remaining outcomes, including metabolic factors and AEs, as secondary outcomes. To accept a review article that is applicable to clinical practice, only those trials with a follow-up period of at least 12 weeks were included. Only articles published in English were considered eligible as the quality of articles published in languages other than English was hard to assess. When several studies from an original study were identified, only the study with the most complete information was included. Studies with at 
least one of the following criteria were excluded: (1) experimental studies, (2) reviews or prevalence studies, (3) studies including type 1 diabetic or non-diabetic patients, (4) patients with fatty liver caused by alcohol or some known agents, and (5) trials that provided insufficient information to enable a judgment. Conference abstracts with sufficient information were considered eligible.

\section{Data Extraction}

Two investigators independently selected the eligible trials according to the selection criteria and extracted the data from each eligible trial with a standard sheet. In this study, the information extracted included the name of the leading author, publication year, country of leading author, characteristics of patients, including sample size, gender, age, details of intervention regimens, and outcomes of interest. For multiple-arm studies, outcome data were collected from all groups that satisfied the inclusion criteria, as recommended in the Cochrane Handbook for Systematic Reviews of Interventions, and reported individually [33]. If the trials presented their continuous variables as means with ranges, then those variables were converted into the mean and variance (standard deviation [SD]) according to the method proposed by Hozo et al. [36]. We contacted the leading author to obtain additional information when essential data could not be extracted from the full-text publication. Any disagreement on the inclusion and extraction of the basic information and data was resolved by consultation with a third author.

\section{Assessment of Risk of Bias}

Our team appointed two independent reviewers to complete the process of appraising the risk of bias of each eligible study using the Cochrane risk of bias assessment tool: method of generating randomization sequence; method of conducting allocation concealment; blinding of participants, personnel, and outcome assessors; completeness of outcome data; selective reporting; and other biases, such as financial support [37]. We further graded the overall methodological quality of each study after determining the consistent level of actual information with assessment criteria [38]. Any conflicts regarding the assessment of risk of bias were resolved by arbitration with a third senior reviewer.

\section{Statistical Analysis}

In this review and meta-analysis, all outcomes were continuous data; therefore, the mean difference (MD) or standard mean difference (SMD) with the 95\% confidence interval (CI) was used. A random-effects model was performed to calculate all estimates because this model considered within- and between-study heterogeneity simultaneously [39]. The Q statistic (significant when $P<0.10$ ) was adopted to describe the heterogeneity qualitatively [40]. The $I^{2}$ statistic (significant when $I^{2}>50 \%$ ) was used to quantitatively estimate the proportion of overall variation [41]. A funnel plot was drawn for a single outcome to qualitatively inspect the publication bias if the accumulated number of included studies was $>10$ [42]. All statistical analyses were completed using RevMan version 5.3 software (Copenhagen, Denmark: The Nordic Cochrane Centre, The Cochrane Collaboration, 2013).

\section{RESULTS}

\section{Identification and Selection of Eligible Studies}

A total of 136 studies were identified after the initial search of the electronic databases. Of these, 36 duplicate studies were excluded after running the 'Find Duplicates' function that is embedded in the EndNote software and subsequently performing a manual check. After careful reviewing of the titles and abstracts of the remaining 100 items, 23 studies were identified, and the full-text of these 23 studies were retrieved to further determine the eligibility of each trial. Of these 23 studies, 12 trials were excluded for the following reasons: reviews 


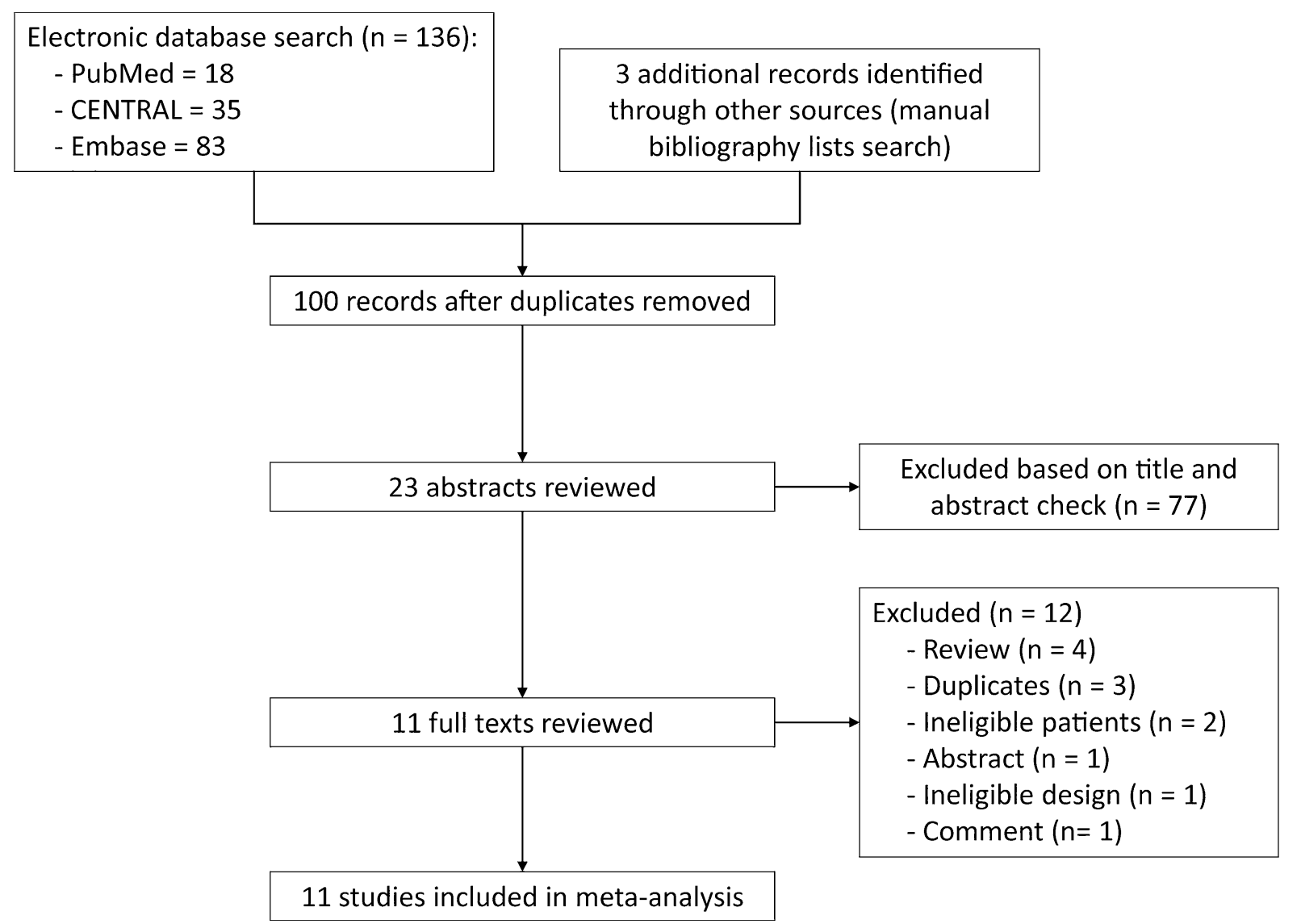

Fig. 1 Flow chart of identification and selection of eligible trials. 'Other sources' refers to bibliographic lists of eligible studies and topic-related reviews

$(n=4)$, duplicate studies $(n=3)$, ineligible patients $(n=2)$, conference abstract $(n=1)$, ineligible design $(n=1)$, and comment $(n=1)$. Ultimately, 11 studies [27-30, 43-48] were included in the quantitative analysis. Figure 1 depicts the study identification and selection of eligible trials.

\section{Details of Characteristics of all Eligible Trials}

The detailed characteristics of all eligible trials are presented in Table 1. All studies [27-30, 43-48] included in the analysis were reported between 2015 and 2020, and the sample size of each trial varied from 14 to 127 , with a median sample size of 48 . The duration of follow-up ranged from 12 weeks to 24 months. All trials [27-30, 43-48] used liraglutide to treat NAFLD in patients with T2DM. However, the control regimens in eligible trials were distinct; of the 11 trials, five used placebo $[27,29,43,45,46]$, one used pioglitazone [48], two used metformin [28, 47], and three used insulin [30, 44, 49].

\section{Assessment of Risk of Bias of Single Trial}

A summary of the information on the methodological quality of each included study is provided in Fig. 2. Among the 11 trials included for the final analysis, eight $[28,30,43-46,49]$ introduced the details of randomization sequence generation, such as computerized randomization sequence and random number table. Appropriate allocation concealment was conducted in six trials $[28,30,43,45,46,49]$. Two trials $[27,30]$ 


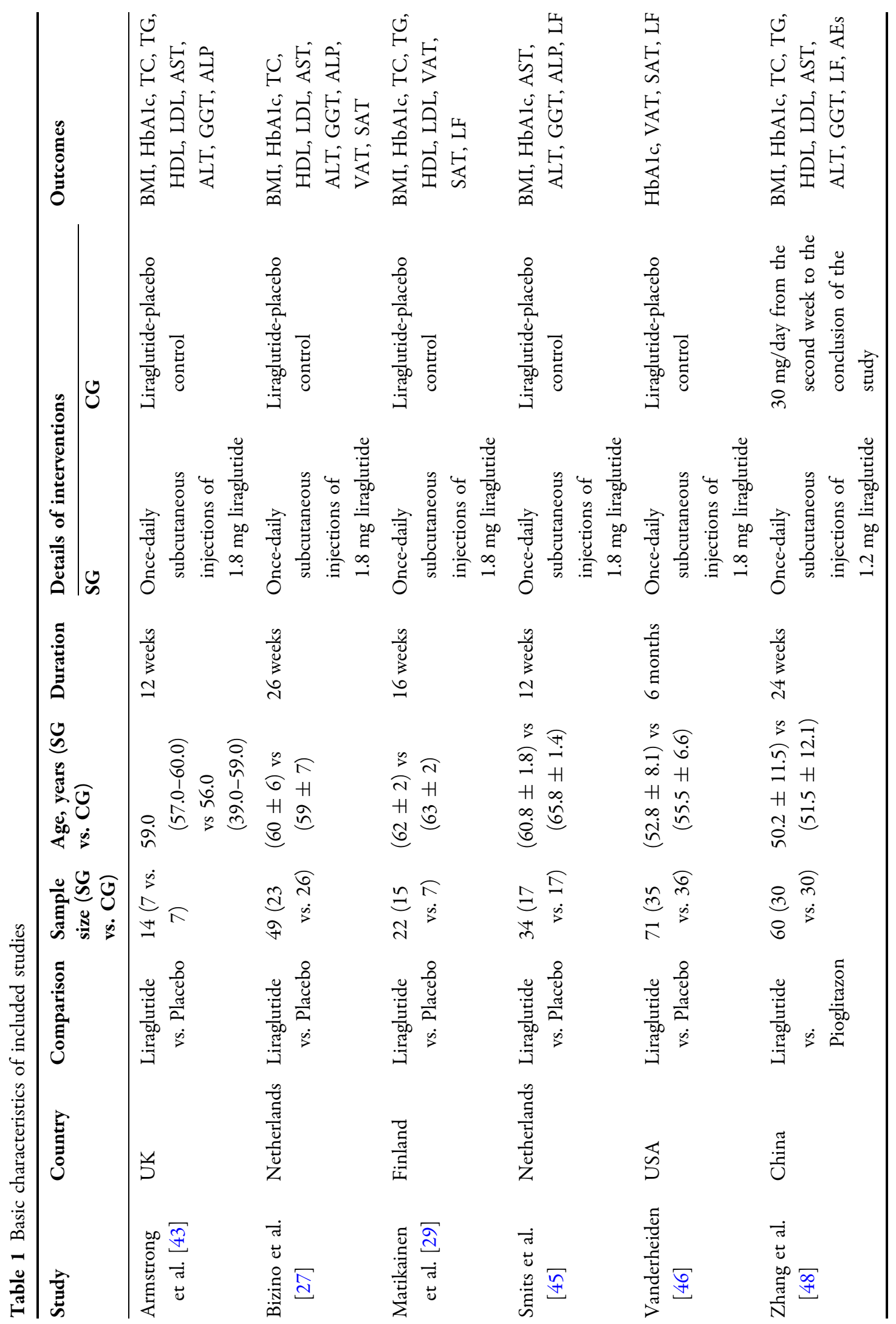




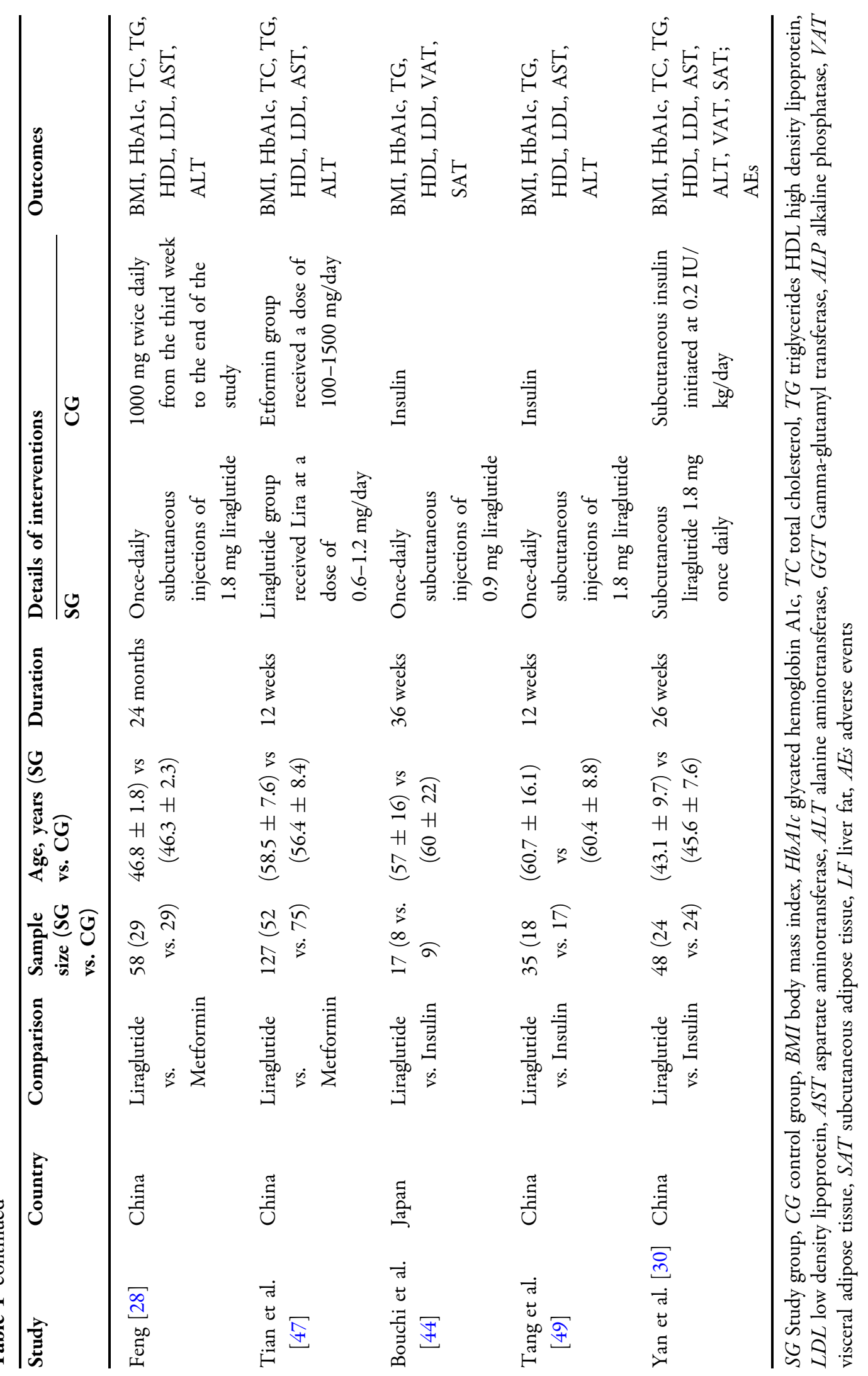




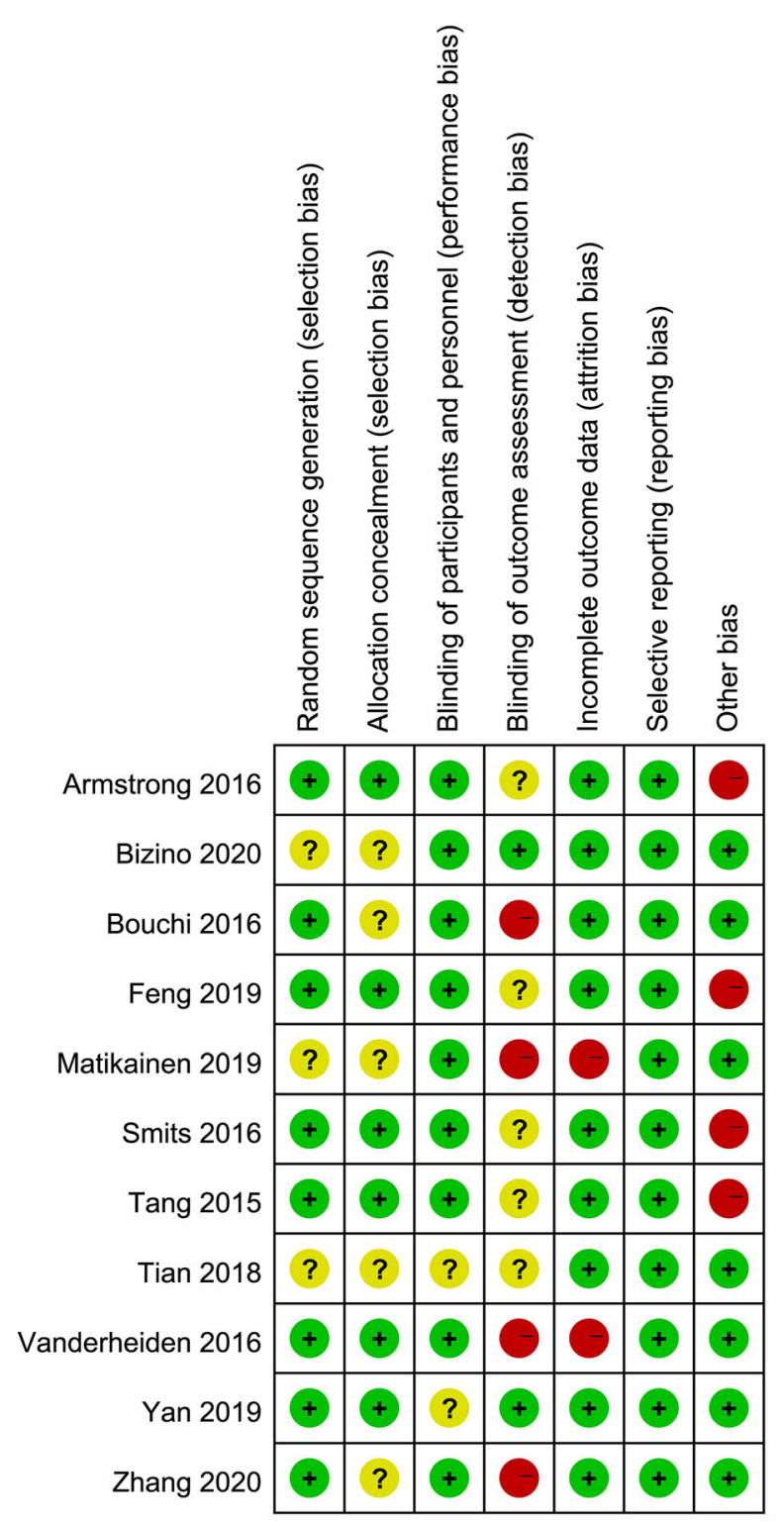

Fig. 2 Risk of bias summary. Green, yellow and red solid circles represent low, unclear, and high risk of bias, respectively

declared that investigators, participants, and outcome assessors were all blinded appropriately. Two trials $[29,46]$ did not report the reasons for drop out. All studies reported anticipating outcomes which were designed in the respective Methods section. Four studies $[28,43,45,49]$ were rated as high risk of bias due to extremely small sample size. In summary, the overall level of quality of all eligible trials was considered to be moderate.

\section{Meta-Analysis of Anticipated Comparisons}

\section{Liraglutide vs. Placebo}

Of the eligible trials, five [27, 29, 43, 45, 46] involving 190 patients compared liraglutide and placebo. The results of the meta-analysis revealed that treatment with liraglutide did not decrease liver enzymes, including AST, ALT, GGT, and ALP (Fig. 3), and LF (three trials; MD $-1.95 ; \quad 95 \%$ CI -4.09 to $0.20 ; \quad P=0.08$; $I^{2}=58 \%$; Fig. 4), adipose tissues, including SAT and VAT (ESM Fig. S1), lipoproteins, including HDL and LDL (ESM Fig. S2), but it did decrease BMI (four trials; MD $-1.13 \mathrm{~kg} / \mathrm{m}^{2} ; 95 \%$ CI -2.03 to $-0.23 ; P=0.01 ; I^{2}=83 \%$; Fig. 3$)$, HbA1c (five trials; MD -0.86 ; $95 \% \mathrm{CI}-1.22$ to $-0.51 ; P<0.001 ; I^{2}=67 \%$; EMS Fig. S3), TC (three trials; MD - $0.34 \mathrm{mmol} / \mathrm{L} ; 95 \% \mathrm{CI}-0.65$ to $-0.03 ; P=0.03 ; I^{2}=28 \%$; EMS Fig. S4), and TG (two trials; MD $-0.29 \mathrm{mmol} / \mathrm{L} ; 95 \% \mathrm{CI}$ -0.57 to $-0.01 ; \quad P=0.04 ; \quad I^{2}=25 \% ; \quad$ EMS Fig. S5).

\section{Liraglutide vs. Pioglitazone}

Of all eligible trials, one trial [48] involving 60 patients compared liraglutide with pioglitazone. The meta-analysis results suggested that liraglutide treatment decreased LF (MD - 2.50; 95\% CI -4.30 to $-0.70 ; P=0.006$; Fig. 4 ) and BMI $\left(\mathrm{MD}-4.10 \mathrm{~kg} / \mathrm{m}^{2} ; 95 \% \mathrm{CI}-6.27\right.$ to $-1.93 ; \quad P<0.001 ;$ Fig. 5), but not liver enzymes, including AST, ALT, and GGT (Fig. 3), and LF (three trials; MD - 1.95; 95\% CI - 4.09 to $0.20 ; P=0.08 ; I^{2}=58 \%$; Fig. 4 ), lipoproteins, including HDL and LDL (ESM Fig. S2), HbA1c (EMS Fig. S3), TC (EMS Fig. S4), and TG (EMS Fig. S5) when compared to pioglitazone.

\section{Liraglutide vs. Metformin}

Among all eligible trials, two trials [28, 47] involving 185 patients compared liraglutide with metformin. The results of the meta-analysis revealed no statistically significant difference between liraglutide and metformin in terms of liver enzymes, including AST and ALT (Fig. 3), lipoproteins, including HDL and LDL (EMS 


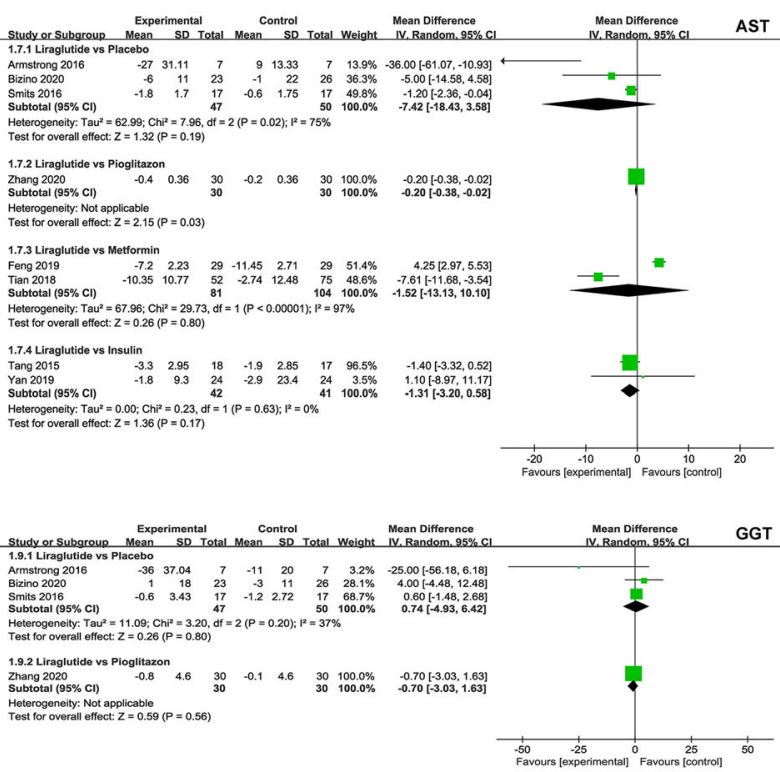

Fig. 3 Meta-analysis of liver enzymes between liraglutide and control groups. The pooled results indicated no significant differences in liver enzymes between liraglutide and control interventions. The summary effect estimates (mean difference $[\mathrm{MD}]$ ) for an individual randomized controlled trial (RCT) are indicated by green rectangles (the size of the rectangle is proportional to the study

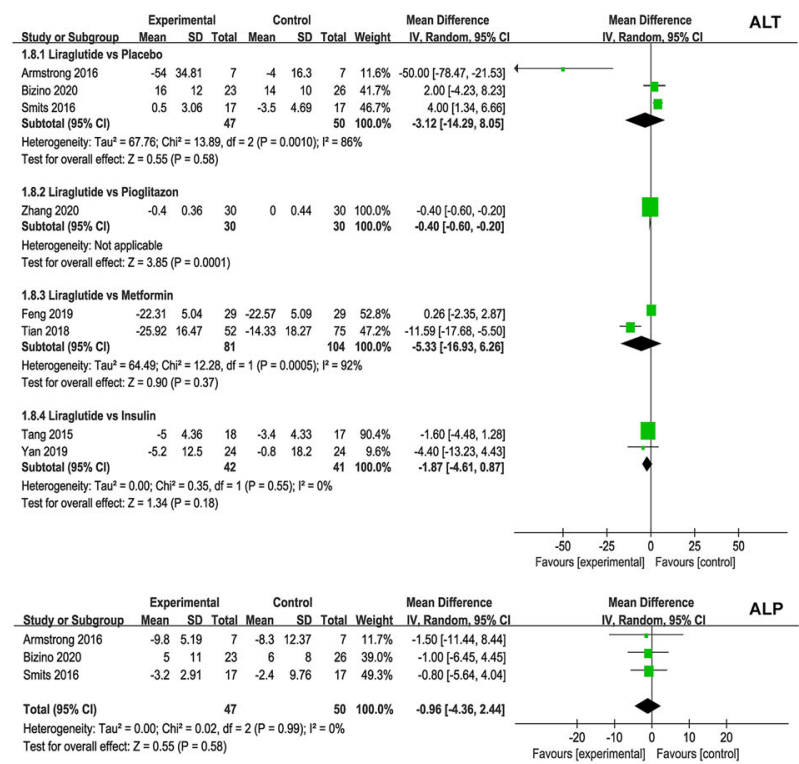

weight), and the black horizontal lines represent the 95\% confidence interval $(C I)$. The overall summary effect estimates (MD) and 95\% CI are indicated by the black diamond. $I V$ Inverse variance, $A S T$ aspartate aminotransferase, $A L T$ alanine aminotransferase, GGT gammaglutamyl transferase, $A L P$ alkaline phosphatase

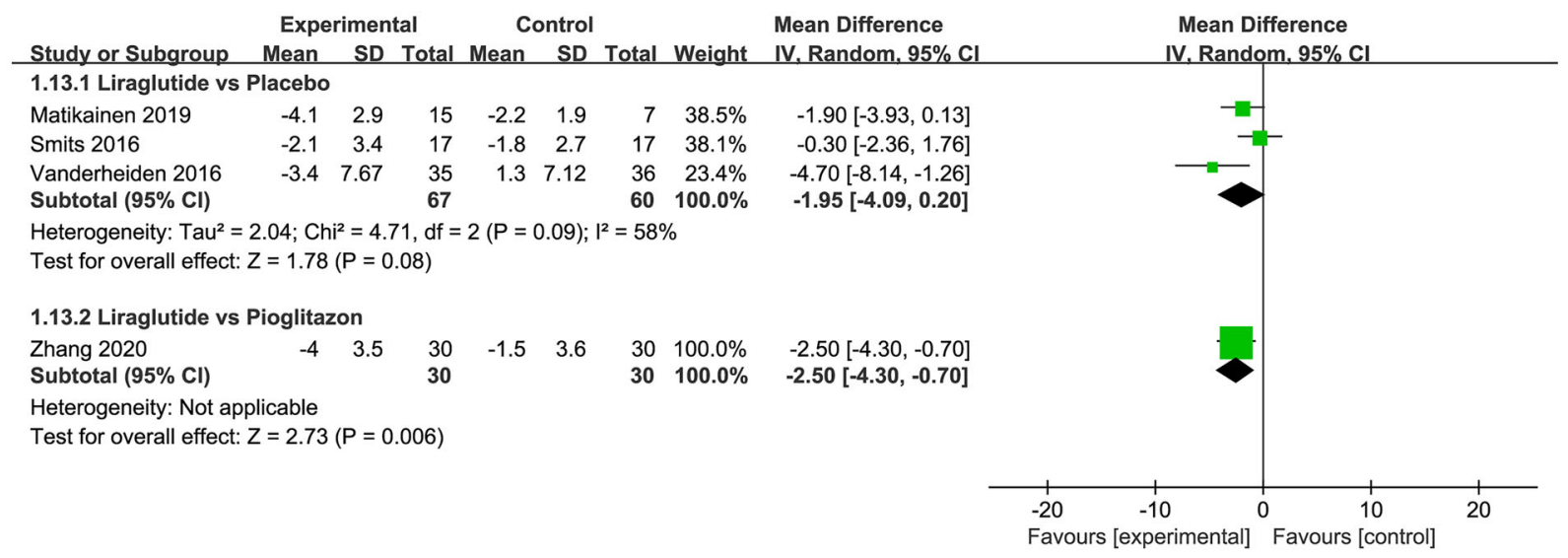

Fig. 4 Meta-analysis of liver fat content between liraglutide and placebo or other active control agents. SD Standard difference

Fig. S2), HbA1c (EMS Fig. S3), and TG (EMS Fig. S5), but the results did shown a significant difference in terms of BMI (two trials; MD $-1.07 \mathrm{~kg} / \mathrm{m}^{2} ; \quad 95 \%$ CI -2.06 to -0.08 ; $P=0.03 ;$ Fig. 5) and TC (two trials; MD
$0.09 \mathrm{mmol} / \mathrm{L} ; 95 \%$ CI $0.01-0.18 ; P=0.04 ; \mathrm{ESM}$ Fig. S4).

\section{Liraglutide vs. Insulin}

Three eligible studies [30, 44, 49] involving 100 patients investigated the comparative efficacy 


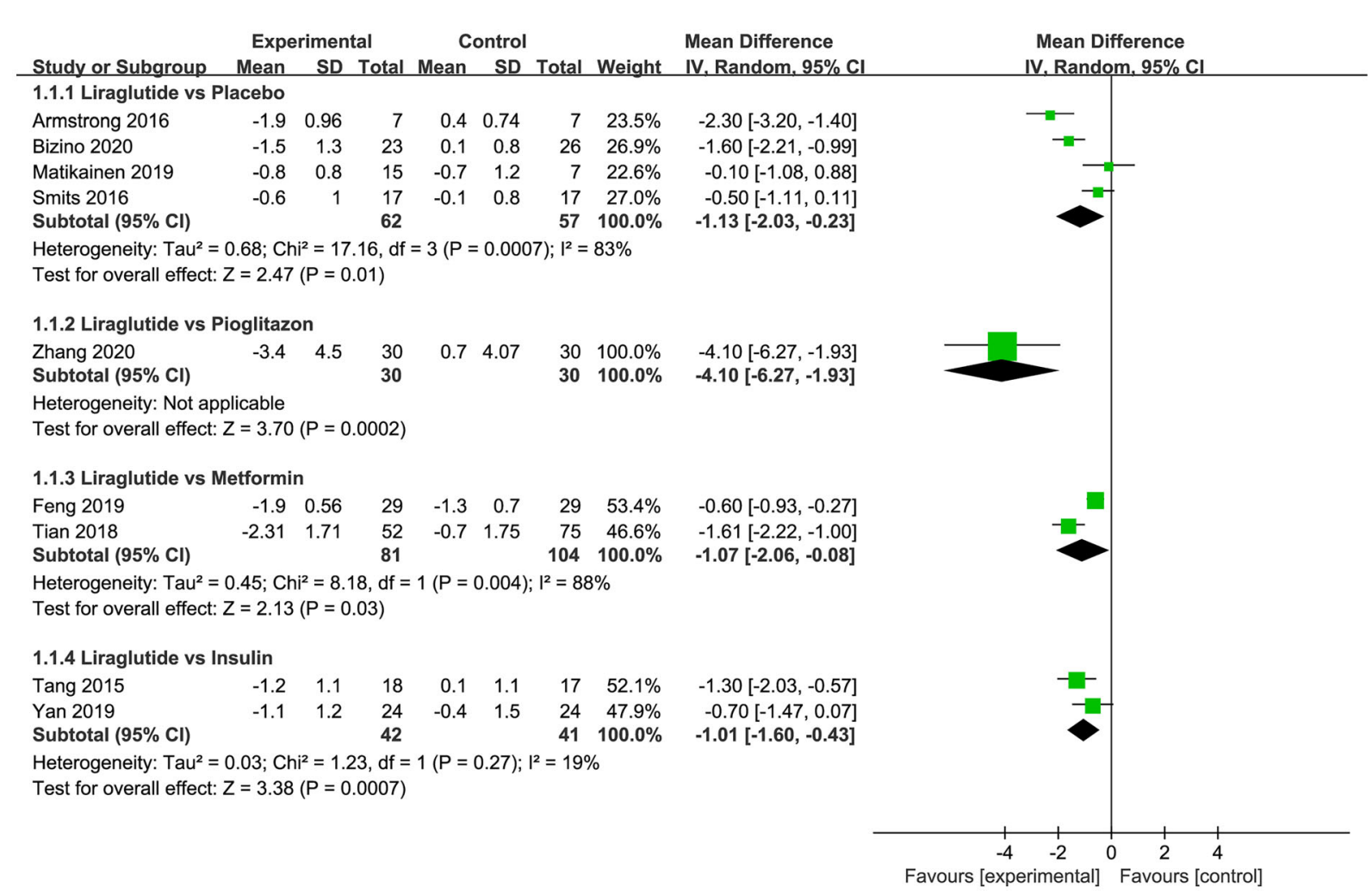

Fig. 5 Meta-analysis of body mass index between liraglutide and placebo or other active control agents

between liraglutide and insulin. The results of the meta-analysis reveaked that liraglutide did not reduce liver enzymes, including AST and ALT (Fig. 3), adipose tissues, including SAT and VAT (EMS Fig. S1), and TC (EMS Fig. S4), but it did reduce BMI (two trials; MD $-1.01 \mathrm{~kg} / \mathrm{m}^{2}$; 95\% CI -1.60 to $-0.43 ; P<0.01 ;$ Fig. 5$)$, lipoproteins, including HDL (three trials; MD $-0.10 \mathrm{mg} / \mathrm{dl} ; \quad 95 \% \quad$ CI -0.15 to -0.05 ; $P<0.001 ; I^{2}=0 \%$; ESM Fig. S2) and LDL (three trials; $\mathrm{MD}-0.26 \mathrm{mg} / \mathrm{dl} ; 95 \% \mathrm{CI}-0.43$ to $-0.10 ; \quad P=0.002 ; \quad I^{2}=20 \% ; \quad$ EMS Fig. S2), HbA1c (three trials; MD $-0.22 ; 95 \%$ CI -0.41 to $-0.04 ; P=0.02 ; I^{2}=0 \%$; EMS Fig. S3), and TG (three trials; MD $-0.80 \mathrm{mmol} / \mathrm{L} ; 95 \% \mathrm{CI}$ -1.03 to $-0.57 ; \quad P<0.001 ; I^{2}=0 \%$ EMS Fig. S5).

\section{Adverse events}

Two of the 11 eligible trials [30, 48] reported adverse events as outcomes. One study [48] that compared liraglutide with pioglitazone reported that patients in the liraglutide group had more gastrointestinal reactions. Another study [30] that was conducted by Yan and colleagues investigated the comparative efficacy and safety between liraglutide and insulin and detected no significant differences in terms of rate of adverse events.

\section{Publication Bias}

The accumulated number of eligible trials for each outcome of interest was $<10$, and so no funnel plot was drawn to check for potential publication bias.

\section{DISCUSSION}

Nonalcoholic fatty liver disease and NASH are the most common complications in patients with T2DM, and are associated with increased liver- and cardiovascular-related morbidity and mortality [50]. No beneficial therapies have yet been approved for the treatment of NAFLD and NASH [51]. However, the search for effective and safe interventions is ongoing, with the ever- 
increasing prevalence of NAFLD in patients with T2DM a major driving factor [48]. GLP-1 receptor agonists (GLP-1 RAs) are the most likely molecules currently being investigated for the treatment of NAFLD in patients with T2DM as these agents have broad-spectrum effects on glucose and lipid metabolism [31]. Several animal studies have revealed that GLP-1 analogs, such as liraglutide, improve hepatic insulin sensitivity and decrease steatosis and fibrosis $[52,53]$. Many human studies have also been conducted to explore the potential of GLP-1 analogs in the treatment of NAFLD in patients with T2DM, but conflicting results have been reported to date $[43,46,49]$.

In this review, the efficacy and safety of liraglutide in the treatment of NAFLD in patients with T2DM were comprehensively evaluated. Our meta-analysis results suggest that liraglutide was associated with decreased BMI, TC, and TG when compared to placebo, decreased LF and BMI when compared to pioglitazone, decreased BMI and TC when compared to metformin, and decreased BMI, HbA1c, lipoproteins (including HDL and LDL), and TG when compared to insulin. Liraglutide might be associated with increased gastrointestinal reactions related to pioglitazone.

To date, four meta-analyses studies $[7,31,32,54]$ have been published that address the comparative efficacy and safety of GLP-1 RAs and placebo or other active agents. A metaanalysis of individual patient data [7] conducted based on the LEAD program revealed that liraglutide $1.8 \mathrm{mg}$ was safe and well tolerated and that it improved liver enzymes in patients with T2DM. Further analysis indicated that this effect was lost after adjusting for weight loss and glycemic control. In the present study, no improvement in liver enzymes was found in the liraglutide group, although decreased weight and $\mathrm{Hb} 1 \mathrm{Ac}$ were detected. More studies should be designed to establish the efficacy of liraglutide in improving the levels of liver enzymes. In 2016, a systematic review and meta-analysis [32] was carried out to investigate the comparative efficacy of anti-diabetic agents on NAFLD patients with T2DM; the results suggested that GLP-1 RAs provided increased benefits in attenuating hepatic fat content.
However, only one eligible study in that metaanalysis considered liraglutide. In comparison, in our meta-analysis we included three eligible studies that investigated the effect of liraglutide on LF, and the results indicated no significant differences between liraglutide and placebo. Our findings should be considered preferentially above those of the earlier meta-analysis due to the larger sample size. Moreover, a beneficial effect was found in the liraglutide group in terms of LF content when compared to pioglitazone. However, this result must be interpreted with caution as only one eligible study was assessed. Portillo-Sanchez and colleagues [31] summarized treatments of NAFLD in patients with T2DM in a narrative review and suggested that liraglutide may have promising impact on this condition, a result that is consistent with our meta-analysis findings. A recent meta-analysis [54] carried out by Dong and colleagues explored the role of GLP-1 RAs in NAFLD. Based on the results, these authors suggested that GLP-1 RAs, including liraglutide, might improve liver histology and reduce aminotransferase levels from baseline. Although this meta-analysis did not include a subgroup analysis to separately investigate the potential of liraglutide in patients with T2DM, these promising findings are mostly consistent with our results.

The present systematic review and metaanalysis included 11 eligible trials with an accumulated sample size of 535 patients to generate relatively robust pooled results. However, there are some limitations to our study that should be acknowledged. The first and foremost is the inclusion of limited number of eligible studies and the extremely small sample size of each individual study, which deeply impairs the robustness and reliability of the summarized results, especially for pioglitazone and metformin. Therefore, the pooled results for these outcomes should be interpreted with caution. Also, more studies with adequate sample size are required to establish the efficacy and safety of liraglutide. Secondly, most of the eligible studies were designed with different duration of follow-up periods. However, further subgroup analysis was not performed due to the limited number of eligible studies. Thus, 
development of further studies with comparable duration of follow-up is warranted. Thirdly, most of the eligible studies did not describe the details of allocation concealment and performed blinding of outcome assessor, negatively affecting the reliability of results. Therefore, future studies with rigorous methodology should be developed to clarify the role of lipoproteins in the treatment of NAFLD in patients with T2DM. Fourthly, the outcomes that can be used to evaluate the liver histology were not used in most of the included studies, such as steatosis, lobular inflammation, and hepatocellular ballooning. Therefore, further studies considering these outcomes are preferred. Fifth, a number of sodium-glucose cotransporter-2 inhibitors have shown efficacy for NAFLD, and we did not investigate the comparative efficacy and safety between liraglutide and SGLT2 inhibitors because of no eligible original study was identified. So, future studies should be performed to bridge this gap. Finally, significant heterogeneity might impair the reliability and robustness of our findings, and so the results should be cautiously interpreted. Therefore, the potential sources that cause heterogeneity with meta-regression when adequate eligible studies were performed should be explored.

\section{CONCLUSIONS}

Based on limited evidence, liraglutide has been suggested to effectively decrease the BMI, HbA1c, lipids, such as TC and TG or LF in T2DM patients complicated with NAFLD when compared to placebo or other active control agents, including poglitazone, metformin, and insulin, indicating the significant therapeutic efficacy of liraglutide. However, future trials with largescale and rigorous methods are warranted to further establish the role of liraglutide in the treatment of NAFLD in patients with T2DM due to limited number of eligible studies currently available and the extremely small sample size of individual studies.

\section{ACKNOWLEDGEMENTS}

Funding. No funding or sponsorship was received for this study or publication of this article. The journal's Rapid Service Fee was funded by the athors.

Authorship. All named authors meet the International Committee of Medical Journal Editors (ICMJE) criteria for authorship for this article, take responsibility for the integrity of the work as a whole, and have given their approval for this version to be published.

Authors' Contributions. T-TS and Y-JJ conceived the study. Y-JJ, FW and Z-LL captured and selected citations. T-TS, Y-JJ and Z-LL designed the data extraction table. Y-JJ and Z-LL extracted data. T-TS, L-PR and Z-LL performed all statistical analyses and prepared the manuscript draft. T-TS and S-CC revised the initial manuscript. FW, T-TS and Y-JJ critically edited language. All authors approved the final version of manuscript.

Disclosures. Tiantian Song, Yujiao Jia, Zelin Li, Fei Wang, Luping Ren and Shuchun Chen have nothing to disclose.

Compliance with Ethics Guidelines. This article is based on previously conducted studies and does not contain any new studies with human participants or animals performed by any of the authors.

Data Availability. The datasets generated during and/or analyzed during the current study are available from the corresponding author on reasonable request.

Open Access. This article is licensed under a Creative Commons Attribution-NonCommercial 4.0 International License, which permits any non-commercial use, sharing, adaptation, distribution and reproduction in any medium or format, as long as you give appropriate credit to the original author(s) and the source, provide a link to the Creative Commons licence, and indicate if changes were made. The images or 
other third party material in this article are included in the article's Creative Commons licence, unless indicated otherwise in a credit line to the material. If material is not included in the article's Creative Commons licence and your intended use is not permitted by statutory regulation or exceeds the permitted use, you will need to obtain permission directly from the copyright holder. To view a copy of this licence, visit http://creativecommons.org/licenses/by$\mathrm{nc} / 4.0 /$.

\section{REFERENCES}

1. DeFronzo RA, Ferrannini E, Groop L, et al. Type 2 diabetes mellitus. Nat Rev Dis Primers. 2015;1: 15019. https://doi.org/10.1038/nrdp.2015.19.

2. Loomba R, Sanyal AJ. The global NAFLD epidemic. Nat Rev Gastroenterol Hepatol. 2013;10(11): 686-90. https://doi.org/10.1038/nrgastro.2013.171.

3. Younossi ZM, Koenig AB, Abdelatif D, Fazel Y, Henry L, Wymer M. Global epidemiology of nonalcoholic fatty liver disease-meta-analytic assessment of prevalence, incidence, and outcomes. Hepatology. 2016;64(1):73-84. https://doi.org/10. 1002/hep.28431.

4. Pettit S, Cresta E, Winkley K, Purssell E, Armes J. Glycaemic control in people with type 2 diabetes mellitus during and after cancer treatment: a systematic review and meta-analysis. PLoS ONE. 2017;12(5):e0176941. https://doi.org/10.1371/ journal.pone.0176941.

5. Silaghi $\mathrm{CA}$, Silaghi $\mathrm{H}$, Crăciun $\mathrm{AE}$, et al. Age, abdominal obesity, and glycated hemoglobin are associated with carotid atherosclerosis in type 2 diabetes patients with nonalcoholic fatty liver disease. Med Ultrason. 2015;17(3):300-7. https://doi. org/10.11152/mu.2013.2066.173.cas.

6. Targher G, Bertolini L, Padovani R, et al. Prevalence of nonalcoholic fatty liver disease and its association with cardiovascular disease among type 2 diabetic patients. Diabetes Care. 2007;30(5):1212-8. https://doi.org/10.2337/dc06-2247.

7. Armstrong MJ, Houlihan DD, Rowe IA, et al. Safety and efficacy of liraglutide in patients with type 2 diabetes and elevated liver enzymes: individual patient data meta-analysis of the LEAD program. Aliment Pharmacol Ther. 2013;37(2):234-42. https://doi.org/10.1111/apt.12149.
8. Farrell GC, Larter CZ. Nonalcoholic fatty liver disease: from steatosis to cirrhosis. Hepatology. 2006;43(2 Suppl 1):S99-s112. https://doi.org/10. 1002/hep.20973.

9. Ballestri S, Mantovani A, Nascimbeni F, Lugari S, Lonardo A. Extra-hepatic manifestations and complications of nonalcoholic fatty liver disease. Future Med Chem. 2019;11(16):2171-92. https://doi.org/ 10.4155/fmc-2019-0003.

10. Ekstedt M, Hagström H, Nasr P, et al. Fibrosis stage is the strongest predictor for disease-specific mortality in NAFLD after up to 33 years of follow-up. Hepatology (Baltimore, MD). 2015;61(5):1547-54. https://doi.org/10.1002/hep.27368.

11. Younossi ZM, Stepanova M, Rafiq N, et al. Pathologic criteria for nonalcoholic steatohepatitis: interprotocol agreement and ability to predict liverrelated mortality. Hepatology. 2011;53(6):1874-82. https://doi.org/10.1002/hep.24268.

12. Nguyen V, George J. Nonalcoholic fatty liver disease management: dietary and lifestyle modifications. Semin Liver Dis. 2015;35(3):318-37. https:// doi.org/10.1055/s-0035-1562950.

13. Aithal GP, Thomas JA, Kaye PV, et al. Randomized, placebo-controlled trial of pioglitazone in nondiabetic subjects with nonalcoholic steatohepatitis. Gastroenterology. 2008;135(4):1176-84. https:// doi.org/10.1053/j.gastro.2008.06.047.

14. Belfort R, Harrison SA, Brown K, et al. A placebocontrolled trial of pioglitazone in subjects with nonalcoholic steatohepatitis. $\mathrm{N}$ Engl J Med. 2006;355(22):2297-307. https://doi.org/10.1056/ NEJMoa060326.

15. Nelson A, Torres DM, Morgan AE, Fincke C, Harrison SA. A pilot study using simvastatin in the treatment of nonalcoholic steatohepatitis: a randomized placebo-controlled trial. J Clin Gastroenterol. 2009;43(10):990-4. https://doi.org/10.1097/ MCG.0b013e31819c392e.

16. Parker HM, Johnson NA, Burdon CA, Cohn JS, O'Connor HT, George J. Omega-3 supplementation and non-alcoholic fatty liver disease: a systematic review and meta-analysis. J Hepatol. 2012;56(4): 944-51. https://doi.org/10.1016/j.jhep.2011.08. 018.

17. Violi F, Cangemi R. Pioglitazone, vitamin E, or placebo for nonalcoholic steatohepatitis. New Eng J Med. 2010;363(12):1185-6. https://doi.org/10. 1056/NEJMc1006581 (author reply 1186).

18. Ratziu V. Pharmacological agents for NASH. Nat Rev Gastroenterol Hepatol. 2013;10(11):676-85. https://doi.org/10.1038/nrgastro.2013.193. 
19. Kheirvari M, Dadkhah Nikroo N, Jaafarinejad $H$, et al. The advantages and disadvantages of sleeve gastrectomy; clinical laboratory to bedside review. Heliyon. 2020;6(2):e03496. https://doi.org/10. 1016/j.heliyon.2020.e03496.

20. Xu G, Song M. Recent advances in the mechanisms underlying the beneficial effects of bariatric and metabolic surgery. Surg Obes Relat Dis. 2021;17(1): 231-8. https://doi.org/10.1016/j.soard.2020.08. 028.

21. Chalasani N, Younossi Z, Lavine JE, et al. The diagnosis and management of nonalcoholic fatty liver disease: practice guidance from the American Association for the Study of Liver Diseases. Hepatology. 2018;67(1):328-57. https://doi.org/10.1002/ hep. 29367.

22. Garber A, Henry R, Ratner R, et al. Liraglutide versus glimepiride monotherapy for type 2 diabetes (LEAD-3 Mono): a randomised, 52-week, phase III, double-blind, parallel-treatment trial. Lancet. 2009;373(9662):473-81. https://doi.org/10.1016/ s0140-6736(08)61246-5.

23. Vilsbøll T, Zdravkovic M, Le-Thi T, et al. Liraglutide, a long-acting human glucagon-like peptide-1 ana$\log$, given as monotherapy significantly improves glycemic control and lowers body weight without risk of hypoglycemia in patients with type 2 diabetes. Diabetes Care. 2007;30(6):1608-10. https:// doi.org/10.2337/dc06-2593.

24. Montanya E, Sesti G. A review of efficacy and safety data regarding the use of liraglutide, a once-daily human glucagon-like peptide 1 analogue, in the treatment of type 2 diabetes mellitus. Clin Ther. 2009;31(11):2472-88. https://doi.org/10.1016/j. clinthera.2009.11.034.

25. Marso SP, Daniels GH, Brown-Frandsen K, et al. Liraglutide and cardiovascular outcomes in type 2 diabetes. N Engl J Med. 2016;375(4):311-22. https://doi.org/10.1056/NEJMoa1603827.

26. Robinson LE, Holt TA, Rees K, Randeva HS, O'Hare JP. Effects of exenatide and liraglutide on heart rate, blood pressure and body weight: systematic review and meta-analysis. BMJ Open. 2013. https://doi. org/10.1136/bmjopen-2012-001986.

27. Bizino MB, Jazet IM, de Heer P, et al. Placebo-controlled randomised trial with liraglutide on magnetic resonance endpoints in individuals with type 2 diabetes: a pre-specified secondary study on ectopic fat accumulation. Diabetologia. 2020;63(1): 65-74. https://doi.org/10.1007/s00125-019-050216.

28. Feng WH, Bi Y, Li P, et al. Effects of liraglutide, metformin and gliclazide on body composition in patients with both type 2 diabetes and non-alcoholic fatty liver disease: a randomized trial. J Diabetes Investig. 2019;10(2):399-407. https://doi.org/ 10.1111/jdi.12888.

29. Matikainen N, Söderlund S, Björnson E, et al. Liraglutide treatment improves postprandial lipid metabolism and cardiometabolic risk factors in humans with adequately controlled type 2 diabetes: a single-centre randomized controlled study. Diabetes Obes Metab. 2019;21(1):84-94. https://doi. org/10.1111/dom.13487.

30. Yan J, Yao B, Kuang H, et al. Liraglutide, sitagliptin, and insulin glargine added to metformin: the effect on body weight and intrahepatic lipid in patients with type 2 diabetes mellitus and nonalcoholic fatty liver disease. Hepatology. 2019;69(6):2414-26. https://doi.org/10.1002/hep.30320.

31. Portillo-Sanchez P, Cusi K. Treatment of nonalcoholic fatty liver disease (NAFLD) in patients with type 2 diabetes mellitus. Clin Diabetes Endocrinol. 2016;2:9. https://doi.org/10.1186/s40842-0160027-7.

32. Tang W, Xu Q, Hong T, et al. Comparative efficacy of anti-diabetic agents on nonalcoholic fatty liver disease in patients with type 2 diabetes mellitus: a systematic review and meta-analysis of randomized and non-randomized studies. Diabetes Metab Res Rev. 2016;32(2):200-16. https://doi.org/10.1002/ dmrr.2713.

33. Higgins JP, Green S. Cochrane handbook for systematic reviews of interventions version 5.0.0. Naunyn Schmiedebergs Arch Exp Pathol Pharmakol. 2008;5(2):S38.

34. Moher D, Liberati A, Tetzlaff J, Altman DG. Preferred reporting items for systematic reviews and meta-analyses: the PRISMA statement. PLoS Med. 2009;6(7):e1000097. https://doi.org/10.1371/ journal.pmed.1000097.

35. Zhang B, Cui X. Effect of continuing nursing on self-care ability of diabetic patients: a meta-analysis. TMR Integr Nurs. 2019;3(2):49-55.

36. Hozo SP, Djulbegovic B, Hozo I. Estimating the mean and variance from the median, range, and the size of a sample. BMC Med Res Methodol. 2005;5: 13. https://doi.org/10.1186/1471-2288-5-13.

37. Higgins JP, Altman DG, Gøtzsche PC, et al. The Cochrane Collaboration's tool for assessing risk of bias in randomised trials. BMJ (Clinical research ed). 2011;343:d5928. https://doi.org/10.1136/bmj. d5928.

38. Huang W-Q, Yang Z, Tang D-X, et al. Pharmacological intervention of traditional Chinese 
medicine for the quality of life in patients with colorectal cancer: a systematic review and metaanalysis. Tradit Med Res. 2018;003(002):95-105.

39. DerSimonian R, Laird N. Meta-analysis in clinical trials revisited. Contemp Clin Trials. 2015;45(Pt A): 139-45. https://doi.org/10.1016/j.cct.2015.09.002.

40. Bowden J, Tierney JF, Copas AJ, Burdett S. Quantifying, displaying and accounting for heterogeneity in the meta-analysis of RCTs using standard and generalised Q statistics. BMC Med Res Methodol. 2011;11:41. https://doi.org/10.1186/1471-2288-1141.

41. Higgins JP, Thompson SG. Quantifying heterogeneity in a meta-analysis. Stat Med. 2002;21(11): 1539-58. https://doi.org/10.1002/sim.1186.

42. Palma Pérez S, Delgado Rodríguez M. Practical considerations on detection of publication bias. Gac Sanit. 2006;20(Suppl 3):10-6. https://doi.org/ $10.1157 / 13101085$.

43. Armstrong MJ, Hull D, Guo K, et al. Glucagon-like peptide 1 decreases lipotoxicity in non-alcoholic steatohepatitis. J Hepatol. 2016;64(2):399-408. https://doi.org/10.1016/j.jhep.2015.08.038.

44. Bouchi R, Nakano Y, Fukuda T, et al. Reduction of visceral fat by liraglutide is associated with ameliorations of hepatic steatosis, albuminuria, and micro-inflammation in type 2 diabetic patients with insulin treatment: a randomized control trial. Endocr J. 2017;64(3):269-81. https://doi.org/10. 1507/endocrj.EJ16-0449.

45. Smits MM, Tonneijck L, Muskiet MH, et al. Twelve week liraglutide or sitagliptin does not affect hepatic fat in type 2 diabetes: a randomised placebocontrolled trial. Diabetologia. 2016;59(12): 2588-93. https://doi.org/10.1007/s00125-0164100-7.

46. Vanderheiden A, Harrison LB, Warshauer JT, et al. Mechanisms of action of liraglutide in patients with type 2 diabetes treated with high-dose insulin. J Clin Endocrinol Metab. 2016;101(4):1798-806. https://doi.org/10.1210/jc.2015-3906.

47. Tian F, Zheng Z, Zhang D, He S, Shen J. Efficacy of liraglutide in treating type 2 diabetes mellitus complicated with non-alcoholic fatty liver disease. 2018. Biosci Rep. https://doi.org/10.1042/ bsr20181304.

48. Zhang LY, Qu XN, Sun ZY, Zhang Y. Effect of liraglutide therapy on serum fetuin $A$ in patients with type 2 diabetes and non-alcoholic fatty liver disease. Clin Res Hepatol Gastroenterol. 2020. https://doi.org/10.1016/j.clinre.2020.01.007.

49. Tang A, Rabasa-Lhoret R, Castel H, et al. Effects of insulin glargine and liraglutide therapy on liver fat as measured by magnetic resonance in patients with type 2 diabetes: a randomized trial. Diabetes Care. 2015;38(7):1339-46. https://doi.org/10.2337/ dc14-2548.

50. Cegla J. Liraglutide safety and efficacy in patients with non-alcoholic steatohepatitis (LEAN): a multicentre, double-blind, randomized, placebo-controlled phase 2 study. Ann Clin Biochem. 2016;53(4):518. https://doi.org/10.1177/ 0004563216648250 .

51. Armstrong MJ, Gaunt P, Aithal GP, et al. Liraglutide safety and efficacy in patients with non-alcoholic steatohepatitis (LEAN): a multicentre, double-blind, randomised, placebo-controlled phase 2 study. Lancet. 2016;387(10019):679-90. https://doi.org/ 10.1016/s0140-6736(15)00803-x.

52. Ben-Shlomo S, Zvibel I, Shnell M, et al. Glucagonlike peptide-1 reduces hepatic lipogenesis via activation of AMP-activated protein kinase. J Hepatol. 2011;54(6):1214-23. https://doi.org/10.1016/j. jhep.2010.09.032.

53. Trevaskis JL, Griffin PS, Wittmer C, et al. Glucagonlike peptide-1 receptor agonism improves metabolic, biochemical, and histopathological indices of nonalcoholic steatohepatitis in mice. Am J Physiol Gastrointest Liver Physiol. 2012;302(8):G762-772. https://doi.org/10.1152/ajpgi.00476.2011.

54. Dong Y, Lv Q, Li S, et al. Efficacy and safety of glucagon-like peptide-1 receptor agonists in nonalcoholic fatty liver disease: a systematic review and meta-analysis. Clin Res Hepatol Gastroenterol. 2017;41(3):284-95. https://doi.org/10.1016/j. clinre.2016.11.009. 